\title{
Obesity - A Matter of Motivation?
}

\section{(ㄷ)(i) (우)}

\author{
Authors \\ Ruth Hanssen ${ }^{1,2}$, Sharmili E Thanarajah'1, 3, Marc Tittgemeyer ${ }^{1,4}$, Jens C. Brüning1, 2, 4
}

\section{Affiliations}

1 Max Planck Institute for Metabolism Research, Cologne, Germany

2 Policlinic for Endocrinology, Diabetology and Preventive Medicine (PEPD), University of Cologne, Faculty of Medicine and University Hospital Cologne, Cologne, Germany

3 Department of Psychiatry, Psychosomatic Medicine and Psychotherapy, University Hospital Frankfurt, Frankfurt am Main, Germany

4 Cluster of Excellence in Cellular Stress Responses in Aging-associated Diseases (CECAD), Cologne, Germany

Key words

Obesity, motivation, GLP-1, Dopaminergic midbrain

received 29.07.2021

revised 01.10 .2021

accepted 26.10.2021

published online 18.02 .2022

Bibliography

Exp Clin Endocrinol Diabetes 2022; 130: 290-295

DOI $10.1055 / a-1749-4852$

ISSN 0947-7349

(C) 2022. The Author(s).

This is an open access article published by Thieme under the terms of the Creative Commons Attribution-NonDerivative-NonCommercial-License, permitting copying and reproduction so long as the original work is given appropriate credit. Contents may not be used for commecial purposes, or adapted, remixed, transformed or built upon. (https://creativecommons. org/licenses/by-nc-nd/4.0/)

Georg Thieme Verlag KG, Rüdigerstraße 14,

70469 Stuttgart, Germany

\section{Correspondence}

Dr. med. Ruth Hanssen

Policlinic for Endocrinology, Diabetology and Preventive

Medicine

University Hospital Cologne

Kerpener Str. 62

50937 Cologne

Germany

Tel.: + 49/221/478 6668

ruth.hanssen@uk-koeln.de

\section{ABSTRACT}

Excessive food intake and reduced physical activity have long been established as primary causes of obesity. However, the underlying mechanisms causing this unhealthy behavior characterized by heightened motivation for food but not for physical effort are unclear. Despite the common unjustified stigmatization that obesity is a result of laziness and lack of discipline, it is becoming increasingly clear that high-fat diet feeding and obesity cause alterations in brain circuits that are critical for the control of motivational behavior.

In this mini-review, we provide a comprehensive overview of incentive motivation, its neural encoding in the dopaminergic mesolimbic system as well as its metabolic modulation with a focus on derangements of incentive motivation in obesity. We further discuss the emerging field of metabolic interventions to counteract motivational deficits and their potential clinical implications.

\section{Introduction}

The growing obesity pandemic is one of the biggest health problems in the 21st century that gives rise to multiple comorbidities such as cancer or neurodegenerative diseases and thus dramatically increases mortality [1-3]. In the western world, excessive food intake beyond physiological needs as well as reduced physical activity are well established as main causes promoting obesity. However, the underlying mechanisms instigating this maladaptive behavior, which disregards or overrides homeostatic needs, are poorly understood. In everyday life, we constantly make decisions and adapt our behavior to our physiological needs and the surrounding environment - e.g., we decide to go to the bakery across the street to get a delicious sandwich avoiding lunch in the inhouse cafeteria. To ensure our physiological homeostasis and to adapt our behavioral responses, our brain constantly integrates information about the metabolic state (e.g., hunger) with external environmental cues (bakery vs. inhouse cafeteria). External cues can comprise varying motivational signals, which include the incentive value of 
an expected reward (delicious sandwich vs. cafeteria lunch) but also the effort required to obtain the reward (distant bakery across the street vs. close inhouse cafeteria). Thus, everyday decisions in favor of or against food intake are based on cost-benefit analyses weighing the potential food reward against the cost of spending effort to obtain it.

The incentive theory of motivation regards motivational behavior to mainly depend on anticipated rewards and reinforcement; hence, incentive motivation refers to the processes that translate expected reward into the effort spent to obtain the reward [4-6]. Importantly, the subjective valuation of the magnitude of a reward depends on our internal state; a sandwich is regarded as more valuable in a hungry than a sated state [7]. Consequently, our motivation depends on the capacity of our brain to integrate internal state signals (hunger) with environmental cues (distance to bakery, value of sandwich) to guide our behavior.

There is increasing evidence that high-fat diet consumption and obesity perturbate the underlying neural processes leading to maladaptive behavior and motivational deficits. This mini-review aims to give a short overview of incentive motivation, its neural encoding in the dopaminergic mesolimbic system and its metabolic regulation with a focus on derangements of incentive motivation in obesity as one mechanism underlying excessive food intake and reduced physical activity. We further give an outlook on the emerging field of metabolic interventions to counteract motivational deficits and their potential clinical implications.

\section{Encoding of incentive motivation in the dopaminergic midbrain}

Incentive motivation is encoded by the mesolimbic dopaminergic system. Dopaminergic neurons projecting from the ventral tegmental area (VTA) to the nucleus accumbens (NAc) encode both reward-driven learning and motivation to work for reward $[8,9]$. Learning signals are encoded by phasic dopamine release in the NAc. On the other hand, dopamine release ramps up when we approach a reward reflecting reward expectancy [10]. The amount of dopamine, that is released during this ramping-up phase, provides information about the value of the anticipated reward and motivates the amount of effort required to obtain it [11-13]. While our understanding of the differential functions of dopamine in reward learning and motivation are mainly derived from animal studies, human brain imaging studies support these results, as changes in the activity of the NAc were observed to correlate with the subjective value of rewards and its anticipation $[12,14,15]$. Likewise, human pharmacological intervention studies lowering dopaminergic tone have provided evidence for lower effort spending and motivation [16-18].

\section{Motivation in obesity - inconclusive results}

In obese humans, alterations in the fronto-mesolimbic dopamine system (in particular within the dopaminergic projections from the VTA to the NAc) are related to an impaired reward system [19, 20]. A high-fat diet - interestingly even in the absence of obesity - induces downregulation of dopaminergic D2 receptors as described in animal studies [21,22]. On a functional level, high-fat diet consumption also causes a devaluation of standard chow diet, which is encoded by reduced dopamine release from the VTA upon receival of standard food (amongst encoding by hypothalamic agouti-related peptide neurons), and thus diminishes the rewarding properties of food discovery [20]. In humans, the direct impact of a high-fat diet on the mesolimbic system in the absence of obesity has not been studied yet. The data comparing obese and healthy weight participants is consistent with the abovementioned animal literature. Human positron emission tomography (PET) studies revealed a negative correlation between body mass index (BMI) and striatal D2 receptor density or binding potential in obese and overweight humans $[23,24]$. This reduced binding potential of striatal dopamine receptors seems to be associated with an altered striatal dopaminergic tone leading to an imbalance of anticipation and consumption of food reward $[25,26]$. In comparison to lean individuals, humans with obesity show increased neural activation of the NAc when anticipating a reward but experience less activation of reward circuits from the actual food reward consumption $[23,27]$. These changes in D2 receptor binding potential seem to be partly reversible by bariatric surgery-induced long-term weight loss [28-30]. In animals, bariatric surgery even seems to change the motivation for drug rewards via post-surgical increases in bile acid signaling, which reduces accumbal dopamine [31]. However, only little is known about incentive motivation in obese humans and findings portray a heterogeneous picture of effort spending in obesity.

Mathar et al. [32] assessed motivational differences between lean and obese humans in a cost-benefit decision-making paradigm, in which participants had to exert physical effort on a handgrip to win food and non-food reward. Obese participants were less willing to engage in physical effort in particular for high-caloric sweet snack food. In contrast, Epstein et al. as well as Giesen et al. suggest that obese humans may be willing to invest more effort to obtain high-caloric food than lean individuals $[33,34]$.

\section{Metabolic modulation of the dopaminergic midbrain - animal results}

These studies might rest upon incomplete assumptions about modulatory influences on midbrain dopaminergic function, as VTA dopaminergic neurons are not only involved in reward learning and motivation but are also sensitive to nutritional signals [35], post-ingestive effects of food [36, 37], and metabolic state signaled by peptidergic mediators [38-40]. Many orexigenic agents (such as Ghrelin) and postprandial anorexigenic peptides (such as glucagon-like peptide 1 (GLP-1), insulin or leptin) or their agonists can bind to receptors on dopaminergic neurons of the VTA/Nac [41] and hence modulate dopaminergic performance upon their activation affecting motivational behavior. In line, animal studies reveal that the hunger hormone ghrelin applied directly into the VTA increases dopamine levels in the NAc and thus induces motivational behavior for food rewards [4245]. Correspondingly, in a state of overnight fasting with high endogenous levels of ghrelin, ghrelin receptor blockade in the VTA reduces the motivation to work for food reward $[46,47]$. However, the effect of ghrelin on motivation seems to depend on the surrounding environmental cues. In a classical place preference task in rodents, peripheral injection of ghrelin in the absence of food-induced aversive 
behavior [48], while in the presence of food, ghrelin instigated conditioned place preference $[44,48]$ ) indicating that the interplay of endogenous metabolic signals and environmental perception shapes adaptive motivational behavior.

Contrary to ghrelin, postprandial hormones such as insulin or GLP-1 reduce motivated behavior for food rewards in mice by downregulating dopaminergic transmission [49]. Specifically, insulin action on dopaminergic VTA neurons depresses excitatory synaptic transmission [50], decreases dopamine concentrations by enhancing its clearance [51,52], and reduces dopamine release into the NAC [53]. GLP-1 (and its analogues) reduces phasic dopamine release of VTA dopaminergic neurons in response to rewardindicating cues and attenuates synaptic drive onto mesolimbic dopamine neurons [54-56]. Amylin (and its receptor agonists), also seems to affect dopaminergic neurons in the VTA, reducing phasic dopamine action in the NAc and consequently food intake [57, 58]. Its complete role as a modulator of dopaminergic activity and hence motivation still requires further investigation with first data revealing an attenuating effect of Amylin receptor agonists on the rewarding properties of alcohol [59]. Similarly, the adipocytokine leptin, which circulates in proportion to body fat to signal the repletion of long-term energy stores, expresses its receptor on VTA dopaminergic neurons. However, ablation of these leptin receptors does not alter motivational behavior but increases anxiety-like behavior (as these neurons mainly project to the amygdala, which is highly implicated in anxiety) $[60,61]$. Nonetheless, leptin reduces motivational behavior for food. The mode of action seems to be more indirect, however, with leptin receptor-bearing neurons of the lateral hypothalamus, decreasing mesolimbic dopaminergic function as a consequence of increased dopamine uptake in the NAc [62].

Collectively, the orexigenic peptide ghrelin seems to enhance motivational behavior in rodents by upregulating dopaminergic transmission in the mesolimbic system, whereas postprandial and anorexigenic peptides (such as insulin, GLP-1, leptin, and probably amylin) have the opposite effect on dopaminergic function and motivation[63]. However, the food itself, which is used as a reinforcer in motivational paradigms, exerts a time-dependent effect on dopamine release with an immediate orosensory and delayed post-ingestive dopaminergic response [37]. Considering the multitude and complexity of modulatory influences on the dopaminergic mesolimbic system, the above-portrayed roles of peptidergic hormones in the regulation of motivation might be multifaceted with varying effects depending on nutritional/metabolic state.

\section{Metabolic modulation of motivational behavior in humans and its derangements in obesity}

While the modulatory effect of metabolic peptides on the dopaminergic neurocircuitry and motivational behavior is well documented in rodents, evidence for a modulatory role of peripheral peptides or metabolic state affecting motivational behavior in humans is scarce. We, therefore, set up a randomized, placebo-controlled study to assess the effect of GLP-1 on motivation in lean and obese individuals while capturing their metabolic state, i.e., their hunger level and insulin sensitivity. Participants exerted force on a hand-grip to win food and monetary rewards. We could show that hunger increases incentive motivation in lean humans but not in obese humans indicating that motivational irregularities in obesity are state-dependent. We further observed that the effect of hunger on incentive motivation is modulated by the peripheral insulin sensitivity of the individual with impaired peripheral insulin sensitivity reducing the motivational effect of hunger [64]. These results are in line with previous studies showing that altered insulin sensitivity impacts dopaminergic projections of the midbrain and denote a dysfunctional integration of metabolic signals and external cues within the mesolimbic system as the foundation of impaired motivational drive in obesity [65]. The aforementioned heterogeneous results about incentive motivation in obese humans showing both increased and decreased effort spending for rewards might thus be a consequence of neglecting metabolic state, in particular, insulin sensitivity and fasting time/hunger. We further demonstrate that administration of the GLP-1 analogue liraglutide normalizes the motivational effect of hunger in insulin-resistant humans. Most importantly, this holds true for both food and monetary reward, indicating that the modulatory effect of GLP-1 on motivational behavior exceeds a mere food scenario and might prove beneficial in other disorders with motivational deficits [64].

\section{Outlook: Metabolic treatment of motivational deficits in psychiatric diseases}

As GLP-1 normalized motivation in insulin-resistant humans but did not affect motivation in insulin sensitive participants, GLP1 (analogues) might comprise therapeutic potential for motivational dysfunctions in dopaminergic disorders, which are associated with metabolic impairments such as insulin resistance. Insulin resistance is a shared abnormality among many patients with type 2 diabetes mellitus and major depression [66] hence, GLP-1 could be cautiously hypothesized to improve dopaminergic functioning in depression and hence depressive symptom burden in patients suffering from both depression and insulin resistance. Furthermore, GLP-1 receptor polymorphism has been associated with anhedonia - the lack of motivation, which is a core symptom of depression. In a first meta-analysis, treatment of diabetic patients with GLP-1 analogues resulted in a significant reduction of depression scores [67]. However, this meta-analysis is based on eight publications only with heterogeneous cohorts so that the result must be interpreted with caution. While the GLP-1 analogue Liraglutide is already approved as weight loss medication in Germany [68], randomized controlled clinical trials on the efficacy and safety of GLP-1 analogues as a treatment for motivational deficits in diabetic or insulin-resistant patients suffering from depression are lacking.

In animal studies, GLP-1 also reduces the reinforcing efficacy of drugs of abuse so that a potential therapeutic benefit of GLP-1 analogues could also be suspected for detoxification therapies [69]. For instance, GLP-1 analogues were shown to reduce cocaine, amphetamine, alcohol, and nicotine use in animals [70]. Interventional studies in humans are fervently awaited. 

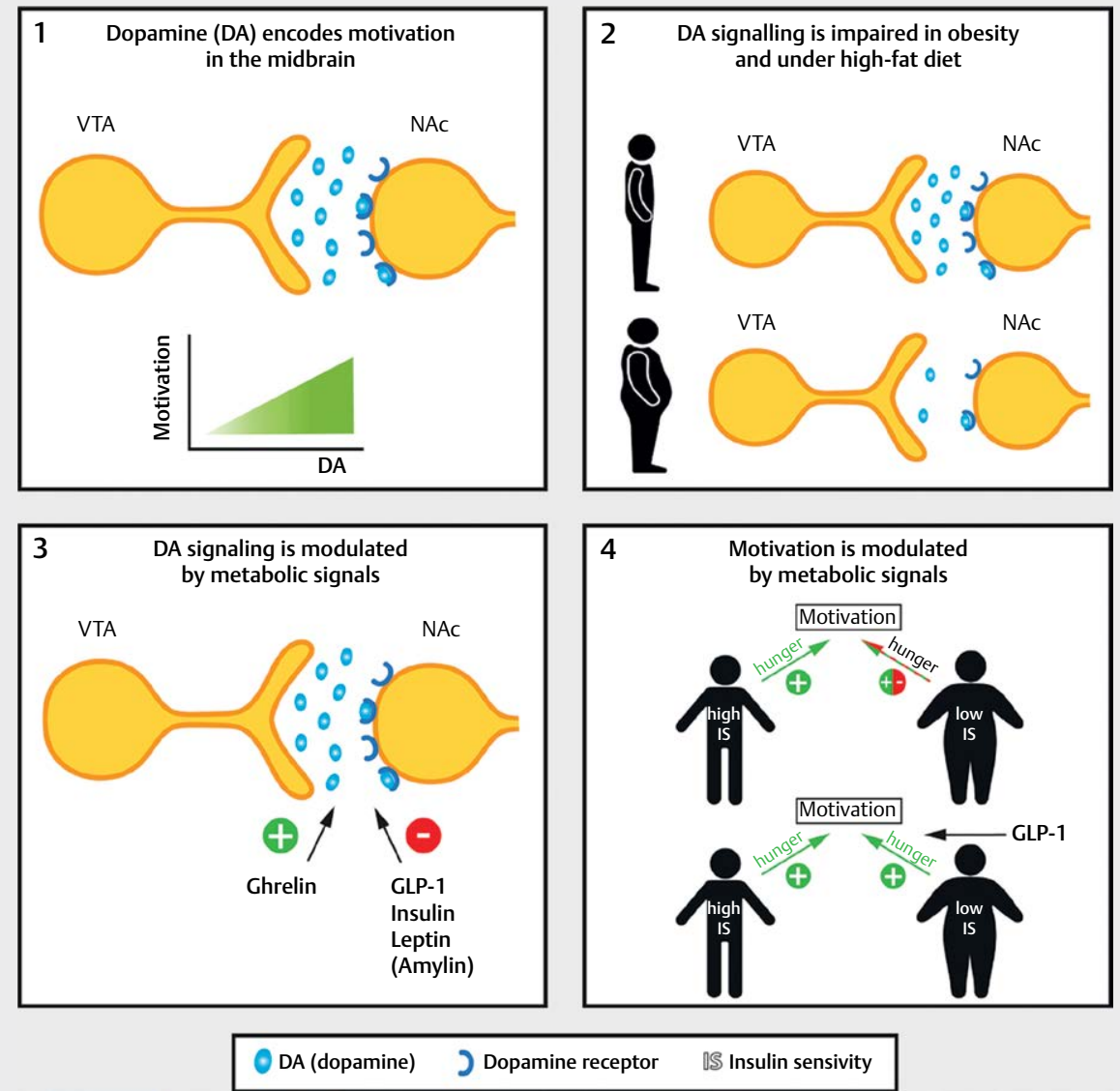

- Fig. 1 Encoding of motivation in the dopaminergic midbrain. Notes. 1) Dopamine (DA) encodes motivation in the midbrain; dopaminergic neurons project from the ventral tegmental area (VTA) to the nucleus accumbens (NAc) with higher dopaminergic tone encoding higher motivation. 2) Dopamine signaling is impaired in obesity and under high-fat diet. The binding potential of dopaminergic D2 receptors is reduced in obesity and high-fat diet (in animal studies) causing a reduced dopamine release from the VTA upon receival of standard food and thus diminishing the rewarding properties of food discovery. 3) Dopamine signaling is modulated by metabolic signals as shown in animal studies. Ghrelin applied into the VTA increases dopamine levels in the NAc and hence motivation; glucagon-like peptide 1 (GLP-1), insulin, leptin and (probably) amylin reduce motivated behavior for food rewards in mice by downregulating dopaminergic transmission. 4) In humans, motivation increases with increasing hunger levels in normal weight humans with good insulin sensitivity, while in obese humans with reduced insulin sensitivity hunger does not affect motivation. Intervention with GLP-1 does not affect motivation in insulin sensitive humans but normalizes the effect of hunger on motivation in insulin resistant humans.

\section{Summary}

In summary, both external cues and internal state signals are integrated into the dopaminergic mesolimbic system to guide our everyday motivational behavior (see $>$ Fig. 1). Dopamine release in the NAc ramps up as a reward approaches, encoding reward expectancy. Metabolic peptide hormones - such as insulin, GLP-1, leptin, or ghrelin - modulate dopaminergic transmission thus regulating motivational behavior, that is, hunger augments motivation to exert effort for rewards. In obesity, insulin resistance reduces the motivational effect of hunger, indicating that a dysfunctional integration of metabolic signals with external cues seems to lead to derangements of incentive motivation representing one possible mechanism underlying excessive food intake and reduced physical activity in obesity. Concomitantly, interventions with metabolic messengers offer new opportunities to reverse motivational deficits. In obese humans, for example, intervention with GLP-1 normalizes motivational behavior.
Future clinical research directions should therefore include the safety and efficacy of clinical interventions with metabolic mediators in pathologies associated with motivational insufficiencies.

\section{Conflict of interest}

The authors declare that they have no conflict of interest.

\section{References}

[1] Bhaskaran K, Douglas I, Forbes $\mathrm{H}$ et al. Body-mass index and risk of 22 specific cancers: A population-based cohort study of 524 million UK adults. Lancet 2014; 384: 755-765

[2] Flegal KM, Kit BK, Orpana $\mathrm{H}$ et al. Association of all-cause mortality with overweight and obesity using standard body mass index categories: A systematic review and meta-analysis. JAMA 2013; 309: $71-82$ 
[3] Bhaskaran K, Dos-Santos-Silva I, Leon DA et al. Association of BMI with overall and cause-specific mortality: A population-based cohort study of 36 million adults in the UK. Lancet Diabetes Endocrinol 2018; 6: 944-953

[4] Killeen PR. Incentive theory. Nebraska Symp Motiv 1981; 29: 169-216

[5] De Houwer ], Hughes S. The psychology of learning: An introduction from a functional-cognitive perspective. MPI Press 2020

[6] Berridge KC. Motivation concepts in behavioral neuroscience. Physiol Behav 2004; 81: 179-209

[7] De Araujo IE, Schatzker M, Small DM. Rethinking food reward. Annu Rev Psychol 2019; 71: 139-164

[8] Fiorillo CD, Tobler PN, Schultz W. Discrete coding of reward probability and uncertainty by dopamine neurons. Science 2003; 299: 1898-1902

[9] Tobler PN, Fiorillo CD, Schultz W. Adaptive coding of reward value by dopamine neurons. Science 2005; 307: 1642-1645

[10] Mohebi A, Pettibone JR, Hamid AA et al. Dissociable dopamine dynamics for learning and motivation. Nature 2019; 570: 65-70

[11] van Swieten MMH, Bogacz R. Modeling the effects of motivation on choice and learning in the basal ganglia. Plos Comput Biol 2020; 16 : e1007465

[12] Bartra O, McGuire JT, Kable JW. The valuation system: A coordinatebased meta-analysis of BOLD fMRI experiments examining neural correlates of subjective value. Neuroimage 2013; 76: 412-427

[13] Howe MW, Tierney PL, Sandberg SG et al. Prolonged dopamine signalling in striatum signals proximity and value of distant rewards. Nature 2013; 500: 575-579

[14] Knutson B, Greer SM. Anticipatory affect: Neural correlates and consequences for choice. Philos Trans R Soc Lond B Biol Sci 2008; 363: 3771-3786

[15] Berke JD. What does dopamine mean? Nat Neurosci 2018; 21: 787-793

[16] Salamone JD, Correa M, Yang JH et al. Dopamine, effort-based choice, and behavioral economics: Basic and translational research. Front Behav Neurosci 2018; 12: 52

[17] Chong TT, Bonnelle V, Manohar S et al. Dopamine enhances willingness to exert effort for reward in Parkinson's disease. Cortex 2015; 69: 40-46

[18] Le Bouc R, Rigoux L, Schmidt L et al. Computational dissection of dopamine motor and motivational functions in humans. J Neurosci 2016; 36: 6623-6633

[19] Kenny PJ. Reward mechanisms in obesity: New insights and future directions. Neuron 2011; 69: 664-679

[20] Mazzone CM, Liang-Guallpa ], Li C et al. High-fat food biases hypothalamic and mesolimbic expression of consummatory drives. Nat Neurosci 2020; 23: 1253-1266

[21] Adams WK, Sussman JL, Kaur S et al. Long-term, calorie-restricted intake of a high-fat diet in rats reduces impulse control and ventral striatal D2 receptor signalling - two markers of addiction vulnerability. Eur J Neurosci 2015; 42: 3095-3104

[22] van de Giessen E, la Fleur SE, Eggels L et al. High fat/carbohydrate ratio but not total energy intake induces lower striatal dopamine D2/3 receptor availability in diet-induced obesity. Int J Obes 2013; 37 : 754-757

[23] Wang G], Volkow ND, Logan J et al. Brain dopamine and obesity. Lancet 2001; 357: 354-357

[24] Haltia LT, Rinne JO, Merisaari $\mathrm{H}$ et al. Effects of intravenous glucose on dopaminergic function in the human brain in vivo. Synapse 2007; 61: 748-756

[25] Stice E, Spoor S, Bohon C et al. Relation of reward from food intake and anticipated food intake to obesity: A functional magnetic resonance imaging study. J Abnorm Psychol 2008; 117: 924-935
[26] Volkow ND, Wang G], Baler RD. Reward, dopamine and the control of food intake: Implications for obesity. Trends Cogn Sci 2011; 15: 37-46

[27] van Bloemendaal L, Veltman DJ, Ten Kulve JS et al. Brain reward-system activation in response to anticipation and consumption of palatable food is altered by glucagon-like peptide- 1 receptor activation in humans. Diabetes Obes Metab 2015; 17: 878-886

[28] van der Zwaal EM, de Weijer BA, van de Giessen EM et al. Striatal dopamine D2/3 receptor availability increases after long-term bariatric surgery-induced weight loss. Eur Neuropsychopharmacol 2016; 26 : 1190-1200

[29] de Weijer BA, van de Giessen E, Janssen I et al. Striatal dopamine receptor binding in morbidly obese women before and after gastric bypass surgery and its relationship with insulin sensitivity. Diabetologia 2014; 57: 1078-1080

[30] Steele KE, Prokopowicz GP, Schweitzer MA et al. Alterations of central dopamine receptors before and after gastric bypass surgery. Obes Surg 2010; 20: 369-374

[31] Reddy IA, Smith NK, Erreger K et al. Bile diversion, a bariatric surgery, and bile acid signaling reduce central cocaine reward. PLoS Biol 2018 16: e2006682

[32] Mathar D, Horstmann A, Pleger B et al. Is it worth the effort? Novel insights into obesity-associated alterations in cost-benefit decisionmaking. Front Behav Neurosci 2015; 9: 360

[33] Epstein LH, Temple JL, Neaderhiser BJ et al. Food reinforcement, the dopamine D2 receptor genotype, and energy intake in obese and nonobese humans. Behav Neurosci 2007; 121: 877-886

[34] Giesen JC, Havermans RC, Douven A et al. Will work for snack food: The association of BMI and snack reinforcement. Obesity (Silver Spring, Md) 2010; 18: 966-970

[35] McCutcheon JE. The role of dopamine in the pursuit of nutritional value. Physiol Behav 2015; 152: 408-415

[36] Fernandes AB, Alves da Silva J, Almeida J et al. Postingestive modulation of food seeking depends on vagus-mdiated dopamine neuron activity. Neuron 2020; 106: 778-788. e6

[37] Edwin TS, Backes H, DiFeliceantonio AG et al. Food intake recruits orosensory and post-ingestive dopaminergic circuits to affect eating desire in humans. Cell Metab 2019; 29: 695-706. e4

[38] Naef L, Pitman KA, Borgland SL. Mesolimbic dopamine and its neuromodulators in obesity and binge eating. CNS Spectrum 2015; 20: $574-583$

[39] Fulton S, Pissios P, Manchon RP et al. Leptin regulation of the mesoaccumbens dopamine pathway. Neuron 2006; 51: 811-822

[40] Liu S, Borgland SL. Regulation of the mesolimbic dopamine circuit by feeding peptides. Neuroscience 2015; 289: 19-42

[41] Figlewicz DP. Expression of receptors for insulin and leptin in the ventral tegmental area/substantia nigra (VTA/SN) of the rat: Historical perspective. Brain Res 2016; 1645: 68-70

[42] Abizaid A, Gao Q, Horvath TL. Thoughts for food: Brain mechanisms and peripheral energy balance. Neuron 2006; 51: 691-702

[43] Al Massadi O, Nogueiras R, Dieguez C et al. Ghrelin and food reward. Neuropharmacology 2019; 148: 131-138

[44] Jerlhag E. Systemic administration of ghrelin induces conditioned place preference and stimulates accumbal dopamine. Addict Biol 2008; 13 : 358-363

[45] Jerlhag E, Egecioglu E, Dickson SL et al. Ghrelin administration into tegmental areas stimulates locomotor activity and increases extracellular concentration of dopamine in the nucleus accumbens. Addict Biol 2007; 12: 6-16

[46] Skibicka KP, Hansson C, Alvarez-Crespo M et al. Ghrelin directly targets the ventral tegmental area to increase food motivation. Neuroscience 2011; 180: 129-137 
[47] Jerlhag E, Janson AC, Waters S et al. Concomitant release of ventral tegmental acetylcholine and accumbal dopamine by ghrelin in rats. PLoS One 2012; 7: e49557

[48] Lockie SH, Dinan T, Lawrence A] et al. Diet-induced obesity causes ghrelin resistance in reward processing tasks. Psychoneuroendocrinology 2015; 62: 114-120

[49] Kleinridders A, Pothos EN. Impact of brain insulin signaling on dopamine function, food intake, reward, and emotional behavior. Curr Nutr Rep 2019; 8: 83-91

[50] Labouèbe G, Liu S, Dias C et al. Insulin induces long-term depression of ventral tegmental area dopamine neurons via endocannabinoids. Nat Neurosci 2013; 16: 300-308

[51] Figlewicz DP, Szot P, Chavez $M$ et al. Intraventricular insulin increases dopamine transporter mRNA in rat VTA/substantia nigra. Brain Res 1994; 644: 331-334

[52] Mebel DM, Wong JC, Dong Y] et al. Insulin in the ventral tegmental area reduces hedonic feeding and suppresses dopamine concentration via increased reuptake. Eur J Neurosci 2012; 36: 2336-2346

[53] Naef L, Seabrook L, Hsiao J et al. Insulin in the ventral tegmental area reduces cocaine-evoked dopamine in the nucleus accumbens in vivo. Eur J Neurosci 2019; 50: 2146-2155

[54] Wang XF, Liu J], Xia J et al. Endogenous glucagon-like peptide-1 suppresses high-fat food intake by reducing synaptic drive onto mesolimbic dopamine neurons. Cell Rep 2015; 12: 726-733

[55] Dickson SL, Shirazi RH, Hansson C et al. The glucagon-like peptide 1 (GLP-1) analogue, exendin-4, decreases the rewarding value of food: A new role for mesolimbic GLP-1 receptors. J Neurosci 2012; 32: 4812-4820

[56] Konanur VR, Hsu TM, Kanoski SE et al. Phasic dopamine responses to a food-predictive cue are suppressed by the glucagon-like peptide-1 receptor agonist exendin-4. Physiol Behav 2020; 215: 112771

[57] Mietlicki-Baase EG, McGrath LE, Koch-Laskowski K et al. Amylin receptor activation in the ventral tegmental area reduces motivated ingestive behavior. Neuropharmacology 2017; 123: 67-79

[58] Mietlicki-Baase EG, Reiner D], Cone JJ et al. Amylin modulates the mesolimbic dopamine system to control energy balance. Neuropsychopharmacology 2015; 40: 372-385

[59] Kalafateli AL, Vallöf D, Jerlhag E. Activation of amylin receptors attenuates alcohol-mediated behaviours in rodents. Addict Biol 2019; 24: 388-402

[60] Liu ], Perez SM, Zhang W et al. Selective deletion of the leptin receptor in dopamine neurons produces anxiogenic-like behavior and increases dopaminergic activity in amygdala. Mol Psychiatry 2011; 16 : 1024-1038
[61] Leshan RL, Opland DM, Louis GW et al. Ventral tegmental area leptin receptor neurons specifically project to and regulate cocaine- and amphetamine-regulated transcript neurons of the extended central amygdala. J Neurosci 2010; 30: 5713-5723

[62] Leinninger GM, Opland DM, Jo YH et al. Leptin action via neurotensin neurons controls orexin, the mesolimbic dopamine system and energy balance. Cell Metab 2011; 14: 313-323

[63] Plassmann H, Schelski DS, Simon MC et al. How we decide what to eat: Toward an interdisciplinary model of gut-brain interactions. Wiley Interdiscip Rev Cogn Sci 2021; e1562

[64] Hanssen R, Kretschmer AC, Rigoux L et al. GLP-1 and hunger modulate incentive motivation depending on insulin sensitivity in humans. Mol Metab 2021; 45: 101163

[65] Edwin Thanarajah S, Iglesias S, Kuzmanovic B et al. Modulation of midbrain neurocircuitry by intranasal insulin. Neuroimage 2019; 194: $120-127$

[66] Hamer JA, Testani D, Mansur RB et al. Brain insulin resistance: A treatment target for cognitive impairment and anhedonia in depression. Exp Neurol 2019; 315: 1-8

[67] Pozzi M, Mazhar F, Peeters G et al. A systematic review of the antidepressant effects of glucagon-like peptide 1 (GLP-1) functional agonists: Further link between metabolism and psychopathology: Special Section on "Translational and Neuroscience Studies in Affective Disorders". Section Editor, Maria Nobile MD, PhD. This Section of JAD focuses on the relevance of translational and neuroscience studies in providing a better understanding of the neural basis of affective disorders. The main aim is to briefly summaries relevant research findings in clinical neuroscience with particular regards to specific innovative topics in mood and anxiety disorders. J Affect Disord 2019; 257: $774-778$

[68] Pi-Sunyer X, Astrup A, Fujioka $\mathrm{K}$ et al. A randomized, controlled trial of $3.0 \mathrm{mg}$ of liraglutide in weight management. N Engl J Med 2015; 373: $11-22$

[69] Schmidt HD, Mietlicki-Baase EG, Ige KY et al. Glucagon-like peptide-1 receptor activation in the ventral tegmental area decreases the reinforcing efficacy of cocaine. Neuropsychopharmacology 2016; 41 : 1917-1928

[70] Eren-Yazicioglu CY, Yigit A, Dogruoz RE et al. Can GLP-1 be a target for reward system related disorders? A qualitative synthesis and systematic review analysis of studies on palatable food, drugs of abuse, and alcohol. Front Behav Neurosci 2020; 14: 614884 